\title{
Tumor chemosensitization by physical exercise? Insights from an animal model
}

\author{
"Physical exercise science is probably coming of age not only in \\ rehabilitation clinical oncology, but also as a potential therapeutic tool.”
}

\section{Mauro Vaccarezza ${ }^{*, 1,2} \&$ Marco Vitale ${ }^{3}$}

The role of physical exercise for the maintenance of human health, for the prevention and cure of metabolic syndrome and diabetes and for the prevention of cardiovascular diseases are widely demonstrated [1]. The major focus in the studies of physical exercise in oncology has been the effect of exercise in to attenuate and improve symptoms outcomes and quality of life: epidemiological data showed a statistically significant inverse ratio between physical activity or recurrence/progression in a variety of cancers [2]. Thus a renowned interest in the effects of physical exercise on cancer biology arises and it is of paramount translational importance. In this regard, a recent provocative set of experiments showed an enhanced tumor perfusion and reduced hypoxia in a model of orthotopic prostate cancer in rats exposed to treadmill exercise [3]. Strikingly, the measurements were performed on the conscious animal, which circumvents the effects of anesthetics on central hemodynamics and tumor blood stream and provide an accurate evaluation of the resting versus exercising condition [3].

The blood flow increase of $200 \%$ as well the $50 \%$ reduction in tumor hypoxia during exercise are significant findings [3] that are of particular interest in view of the driving mechanisms of the metastatic cascade and to improve effectively compound delivery to tumors. First of all, exercise-driven changes in the tumor microenvironment may be a significant mechanism explaining the inverse relationship between physical activity and tumor-specific outcomes [2]. Furthermore, a suggested mechanism is the 'normalization' of the endothelial wall that is impaired in tumors and it represents a daunting barrier to efficient drug delivery $[4,5]$. Tumor blood flow increased and microvessel density increased during exercise whereas tumor hypoxia decreased (hypoxia being an important microenvironment parameter positively linked to tumor aggressiveness, spreading capacity and inversely related to therapeutic response, and radiosensitivity) [4]. Of note tumor microvessels were less prone to vasoconstriction at higher blood pressure during the treadmill activity [3]. This observation could explain the overall enhanced tumor flow during exercise. The biological and translational consequences remain to be determined but this study clearly suggests that exercise can modulate several host and cancer pathways implicated in progression and therapeutic efficacy. Importantly, human biopsy samples of tumors demonstrate similar structural vessel abnormalities to those seen in animal models and human tumors are hypoxic and have

\section{KEYWORDS}

- physical exercise $\bullet$ prostate cancer $\bullet$ tumor perfusion $\bullet$ tumor vascular permeability

\footnotetext{
"The role of physical exercise for the maintenance of human health, for the prevention and cure of metabolic syndrome and diabetes and for the prevention of cardiovascular diseases are widely demonstrated."
}

'School of Biomedical Sciences, Faculty of Medicine \& Biomedical Sciences, University of Queensland, St Lucia Campus, Brisbane, Australia

2Department of Human Sciences, Society \& Health (HSSH), University of Cassino \& Southern Lazio, Cassino, Italy ${ }^{3}$ University of Parma School of Medicine, Department of Biotechnology, Biomedical \& Translational Sciences (S.Bi.Bi.T), Via Gramsci 14, Parma, Italy

*Author for correspondence: m.vaccarezza@uq.edu.au

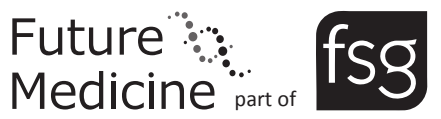


"...physical exercise in selected patients could be an inexpensive and valuable way to enhance drug potency with obvious translational effects in a clinical setting and possibly in cancer survival.” a raised interstitial fluid pressure that hampers drug delivery [4].

Why decreasing hypoxia and increasing blood perfusion is pivotal for improving cancer treatment? The ratio between pro- and anti-angiogenic signaling as well as physical compression leads to abnormal vessels and impaired blood perfusion in malignancies $[4,5]$. The amount of blood flow impairment changes with tumor growth stage and location and can differ among tumor regions or between a primary tumor and its metastases. This progressively worsening heterogeneity in blood perfusion as tumors grow raises an interesting conundrum. If a tumor needs blood vessels to grow and to spread, how does it keep growing when growth impairs the very blood supply that brings the required nutrients and removes waste products? This apparent paradox can be understood by thinking about how a reduced blood supply can impart a survival advantage to these rebel cells by creating an abnormal microenvironment characterized by hypoxia and acidosis. Furthermore, an interesting potential mechanism of detrimental action is that impaired blood supply and the abnormal tumor microenvironment help cancer cells evade the immune system, increase their invasive and metastatic potential, and apply selective survival pressures to which cancer cell populations adapt. Under physiological conditions, immune cells constantly patrol tissues to identify and destroy pathogens, foreign antigens and abnormal cells. However, a hypoxic and acidic microenvironment reprograms the resident macrophages (phagocytes) - whose job is to recognize, engulf and remove dying cells into a protumorigenic and immunosuppressive phenotype [6-12].

Hypoxia and acidosis can also attenuate the killing potential of immune effector cells within the tumor microenvironment. Specifically, growth factors and cytokines (e.g., TGF- $\beta$ and VEGF) induced by hypoxia or acidosis suppress the activity of $T$ lymphocytes and inhibit the ability of dendritic cells to process tumor antigens and present them to lymphocytes [13,14]. In addition, hypoxia can directly upregulate, via HIF1 $\alpha$ activation, the expression of the immune checkpoint protein PD-L1 by myeloid-derived suppressor cells, dendritic cells and cancer cells to aid immune suppression and evasion [15]. Moreover, hypoxia may select for more malignant cells because cells that respond to physiological cues normally undergo apoptosis under hypoxic conditions [12]. Hypoxia can increase the invasive activity of cancer cells by inducing the production of promigratory proteins (e.g., SDF1 $\alpha$ and HGF) and proinvasive extracellular matrix molecules [8,11]. Hypoxia also provides a pabulum for so-called cancer stem cells and facilitates inflammation while also giving resistance to radiation and to many extensively used therapeutic agents [12]. Taken together, these observations explain why intratumoral hypoxia correlates with a poor prognosis in many human cancers [12]. Recently approved immune checkpoint inhibitors have led to unprecedented improvements in overall survival in melanoma patients [16]. However, a subset of patients even in this highly responsive disease does not benefit. Additionally, the first US FDA approved therapeutic vaccine, sipuleucel-T, where autologous dendritic cells are exposed to a fusion protein consisting of GM-CSF and prostatic acidic phosphatase and then infused back into the body, demonstrated a modest survival benefit of a few months. Finally, various vaccine and adoptive T-cell therapies, including with chimeric antibody receptors, have shown promise in various malignancies [17]. Normalizing the tumor microenvironment would improve the outcome of all of these different immune-therapies and potentially allow lowering the dose of immunotherapeutic agents, which, in turn, may decrease their toxicity [18]. As stated earlier, the abnormal microenvironment of tumors helps them evade the immune response through multiple mechanisms, including impairment of lymphocyte infiltration, upregulation of immune checkpoint protein expression via hypoxia, recruitment of Tregs and establishment of an immune-suppressive tumor microenvironment that impairs the function of the resident and transiting immune effector cells [18]. It has been demonstrated that normalizing doses of anti-VEGFR2 antibody can alleviate hypoxia, improve the delivery of immune effector cells into the tumor, convert the immunosuppressive microenvironment of tumors into an immune-stimulatory one and improve survival from a vaccine therapy [18]. Physical exercise could even transiently 'normalize' the abnormal tumor vasculature, resulting in improved blood perfusion. The latter would decrease hypoxia (known to confer resistance to radio-, chemoand immune therapies) and increase drug accessibility. Therefore, therapies delivered during the window of normalization might reach greater efficacy $[4,5]$. The normalized vessels would also resist shedding of cancer cells from the primary tumor, potentially decreasing metastases $[4,5]$. 
As suggested by McCullogh et al. [3], aerobic exercise should be the best enhancer of tumor blood flow; nevertheless, it would be interesting to assess the relative contribution of several types of exercise to these changes in endothelial permeability as well as potential molecular mechanisms (secretion and inhibition of angiogenic factors, modification of shear stress sensors in tumor vasculature), either locally and/or systemically that are favorably modified by physical exercise. More data are definitely needed to support these results. Most preclinical studies report no change in tumor growth rate or a modest to robust decrease in tumor growth during pharmacological driven vascular normalization [4]; formal proof of the same findings for the exercise - induced vascular normalization is lacking, but is worth highlighting that there is no strong association between blood flow or oxygen consumption and cancer growth rate in vivo $[19,20]$.

Accurate measurements of tumor blood perfusion and validated criteria of biomarkers of tumor therapy response via vascular modifications are sorely needed $[4-5,20]$ and will substantially reinforce the provoking findings of this study. In conclusion, physical exercise in selected patients could be an inexpensive and valuable way to enhance drug potency with obvious translational effects in a clinical setting and possibly in cancer survival. Physical exercise science is probably coming of age not only in rehabilitation clinical oncology, but also as a potential therapeutic tool.

\section{Financial \& competing interests disclosure}

The authors have no relevant affiliations or financial involvement with any organization or entity with a financial interest in or financial conflict with the subject matter or materials discussed in the manuscript. This includes employment, consultancies, honoraria, stock ownership or options, expert testimony, grants or patents received or pending, or royalties.

No writing assistance was utilized in the production of this manuscript.

\section{References}

1 Vina J, Sanchis-Gomar F, Martinez-Bello V, Gomez-Cabrera MC. Exercise acts as a drug; the pharmacological benefits of exercise. $B r . J$. Pharm. 167, 1-12 (2012).

2 Ballard-Barbash R, Friedenreich CM, Courneya KS, Siddiqi SM, McTiernan A, Alfano CM. Physical activity, biomarkers, and disease outcomes in cancer survivors: a systematic review. J. Natl Cancer Inst. 104, 815-884 (2012)

3 McCullough DJ, Stabley JN, Siemann DW, Behnke BJ. Modulation of blood flow, hypoxia, and vascular function in orthotopic prostate tumors during exercise. J. Natl Cancer Inst. 106, dju036 (2014).

4 Carmeliet P, Jain RK. Principles and mechanisms of vessel normalization for cancer and other angiogenic diseases. Nat. Rev. Drug Discov. 10, 417-427 (2011).

5 Jain RK. Normalizing tumor microenvironment to treat cancer: bench to bedside to biomarkers. J. Clin. Oncol. 31, 2205-2218 (2013).

6 Casazza A, Di Conza G, Wenes M, Finisguerra V, Deschoemaeker S, Mazzone M. Tumor stroma: a complexity dictated by the hypoxic tumor microenvironment.

Oncogene 33, 1743-1754 (2014).

7 Colegio OR, Chu NQ, Szabo AL et al. Functional polarization of tumour-associated macrophages by tumour-derived lactic acid. Nature 513, 559-563 (2014).

8 Finger EC, Giaccia AJ. Hypoxia, inflammation, and the tumor microenvironment in metastatic disease. Cancer Metastasis Rev. 29, 285-293 (2010).

9 Motz GT, Coukos G. Deciphering and reversing tumor immune suppression. Immunity 39, 61-73 (2013).

10 Noy R, Pollard JW. Tumor-associated macrophages: from mechanisms to therapy. Immunity 41, 49-61 (2014).

11 Semenza GL. Oxygen sensing, hypoxiainducible factors, and disease pathophysiology. Annu. Rev. Pathol. 9, 47-71 (2014).

Wilson WR, Hay MP. Targeting hypoxia in cancer therapy. Nat. Rev. Cancer 11, 393-410 (2011).

13 Barsoum IB, Smallwood CA, Siemens DR, Graham CH. A mechanism of hypoxiamediated escape from adaptive immunity in cancer cells. Cancer Res. 74, 665-674 (2014).

14 Gabrilovich DI, Ostrand-Rosenberg S, Bronte $\mathrm{V}$. Coordinated regulation of myeloid cells by tumours. Nat. Rev. Immunol. 12, 253-268 (2012).

15 Noman MZ, Desantis G, Janji B et al. PD-L1 is a novel direct target of HIF-1a, and its blockade under hypoxia enhanced MDSCmediated T cell activation. J. Exp. Med. 211, 781-790 (2014).

16 Naidoo J, Page DB, Wolchok JD. Immune modulation for cancer therapy. Br. J. Cancer doi:10.1038/bjc.348 (2014) (Epub ahead of print).

17 Fridman WH, Pagès F, Sautès-Fridman C, Galon J. The immune contexture in human tumours: impact on clinical outcome. Nat. Rev. Cancer 12, 298-306 (2012).

18 Huang Y, Goel S, Duda DG, Fukumura D, Jain RK. Vascular normalization as an emerging strategy to enhance cancer immunotherapy. Cancer Res. 73, 2943-2948 (2013).

19 Boucher Y, Lee I, Jain RK. Lack of general correlation between fluid interstitial pressure and oxygen partial pressure in solid tumors. Microvasc. Res. 50, 175-182 (1995).

20 Emblem KE, Farrar CT, Gerstner ER et al. Vessel calibre - a potential MRI biomarker of tumour response in clinical trials. Nat. Rev. Clin. Oncol. 11, 566-584 (2014). 